\title{
Modélisation d'un écosystème lotique pollué par une charge organique : prise en compte de l'hydrodynamique et des mécanismes de transport Modelisation of a lotic ecosystem polluted by a pigsty waste :
hydrodynamic and transport mecanisms
}

\author{
B. Cazelles et D. Fontvieille
}

Volume 2, numéro 2, 1989

URI : https://id.erudit.org/iderudit/705028ar

DOI : https://doi.org/10.7202/705028ar

\section{Aller au sommaire du numéro}

\section{Éditeur(s)}

Université du Québec - INRS-Eau, Terre et Environnement (INRS-ETE)

\section{ISSN}

0992-7158 (imprimé)

1718-8598 (numérique)

Découvrir la revue

\section{Citer cet article}

Cazelles, B. \& Fontvieille, D. (1989). Modélisation d'un écosystème lotique pollué par une charge organique : prise en compte de l'hydrodynamique et des mécanismes de transport. Revue des sciences de l'eau / Journal of Water Science, 2(2), 183-209. https://doi.org/10.7202/705028ar
Résumé de l'article

L'article décrit la partie hydrophysique d'un modèle écologique de simulation des transferts de carbone organique dans un cours d'eau pollué par le rejet d'une porcherie. Cette partie est constituée d'un modèle hydrodynamique inspiré du modèle de Saint-Venant, couplé à un modèle de transport basé sur l'équation classique de convection-diffusion. Ces modèles sont appliqués à un écoulement unidirectionnel, non uniforme et non stationnaire.

Les équations de ces deux modèles sont résolues par une méthode aux différences finies utilisant des schémas implicites. L'ajustement des paramètres est réalisé à partir de résultats d'expériences de traçage à la rhodamine.

Appliqués au carbone organique dissous de l'Albenche, les modèles montrent l'extrême étalement des nuages dû aux seuls phénomènes physiques. L'une des interprétations possibles de l'écart entre les valeurs expérimentales et les valeurs calculées au niveau de la station aval, peut être l'importance de la consommation du carbone par les biocoenoses benthiques. 


\title{
Modélisation d'un écosystème lotique pollué par une charge organique : prise en compte de l'hydrodynamique et des mécanismes de transport
}

\author{
Modelisation of a lotic ecosystem \\ polluted by a pigsty waste : \\ hydrodynamic and transport mecanisms
}

B. CAZELLES \{1\}, D. FONTVIEILLE (2)

RÉSUMÉ

L'article décrit la partie hydroph'sique d'un modèle écologique de simulation des transferts de carbone organique dar:s un cours d'eau pollué par le rejet d'une porcherie. Cette partie est constituée d'un modèle hydrodynamique inspiré du modèle de Saint-Venant, coliplé à un modèle de transport basé sur l'équation classique de convection-diffusior. Ces modèles sont appliqués à un écoulement unidirectionnel, non uniforme et non stationnaire.

Les équations de ces deux modèles sont résolues par une méthode aux différences finies utilisant des schémas implicites.

L'ajustement des paramètres est réalisé à partir de résultats d'expériences de traçage à la rhodamine.

Appliqués au carbone organique dissous de t'Albenche, les modèles montrent l'extrême étalement des nuages dû aux seuts phénomènes physiques. L'une des interprétations possibles de l'écart entre les valeurs expérimentales et les valeurs calculées au niveau de la station aval, peut être l'importance de la consommation du carbone par les biocoenoses benthiques.

Mots clés : cours d'eau, modêle, simulation, hydrodynamique, mécanismes de transport, dispersion longitudinale, auto-épuration.

(1) Anjou-Recherche, BP 76, 78600 Maisons-Laffitte, détaché à l'Université de Savoje.

(2) Laboratoire d'Ecologie, Université de Savoie, BP 1104, 73011 Chambéry et UA CNRS 367, Université Lyon I, 69622 Villeurbanne Cédex. 
SUMMARY

A simulation model. of organic carbon movement was designed for a river polluted by piggery wastes.

The physical dynamics of this model, as described in this paper, include a hydrodynamic component inspired by a Saint Venant's model coupled with a transport model based on the classical advection-diffusion equation. The model is applied to unidirectional, non-uniform, unsteady flow conditions.

The equations were solved by a finite difference method using implicit schemes. The parameters of the equation were adjusted to fit flow conditions determined with a rhodamine dye tracer.

When applied to the dissolved organic carbon transport in the Albenche river, the model showed that the distribution of organic carbon in space and in time was due to physical processes only. The differences in concentration between the observed and the expected values in the downstream station could represent the consumption of benthic biotic organic carbon.

Key-words : stream, model, simulation, hydrodynamic, transport mechanisms, longitudinal dispersion, self-purification.

\section{NOTATiONS}

A : surface mouillée de l'écoulement $\left(\mathrm{L}^{2}\right)$

$B$ : laxgeur de la section de I'écoulement, largeur au miroir (L)

$\mathrm{Ba}$ : biomasse des macroinvertébrés benthiques (M. $L^{-3}$ )

$\mathrm{BF}$ : largeur au fond, largeur au radier (L)

$\mathrm{Bi}$ : biomasse bactérienne ou microbienne (M. $\mathrm{L}^{-3}$ )

$C$ : moyenne spatiale de $c$ ou concentration en substrat organique $\left(M . L^{-3}\right.$ )

cpbi : concentration en substrat organique particulaire dans le compartiment benthique (M.L ${ }^{-2}$ )

Cpsi : concentration en substrat organique particulaire en suspension (M. $\left.\mathrm{L}^{-3}\right)$

$\mathrm{Cq}$ : concentration en matière organique dissoute des apports (M. $\mathrm{L}^{-3}$ )

$c$ : concentration moyenne d'une substance $\left(M \cdot \mathrm{L}^{-3}\right.$ )

ca, cai : coefficient de la relation débit-suxface mouillée

$c q$ : coefficient de la relation débit-débit d'apport

$\mathrm{D}_{\mathrm{L}}$ : coefficient de dispersion longitudinale $\left(\mathrm{L}^{2} \cdot \mathrm{T}^{-1}\right)$

$d$ : longueur du tronçon étudié $(\mathrm{L})$

ea, eai : coefficient de la relation débit-surface mouillée

eq : coefficient de la relation débit-débit d'apport 
$\mathrm{g}$ : accélération de la pesanteur (L.. ${ }^{-2}$ )

$H$ : hauteur d'eau (L)

$\mathrm{Hm}$ : hauteur d'eau moyenne (L)

I : pente moyenne du cours d'eau

$i$ : indice indiquant soit la classe de taille des particules organiques, soit le numéro du tronçon considéré

$\mathrm{J}$ : coefficient. de perte de charge

Kd : taux de dégradation de la rhodamine $\left(\mathrm{T}^{-1}\right)$

Kxi : terseur du coefficient de diffusion turbulente $\left(\mathrm{L}^{2} \cdot \mathrm{T}^{-1}\right)$

$k$ : coefficient de frottement

$\mathrm{Pm}$ : périmètre moujillé (L)

$Q$ : dêbit $\left(\mathrm{L}^{3} \cdot \mathrm{T}^{-1}\right)$

$\mathrm{q}$ : débit $\mathrm{d}^{\prime}$ apport $\left(\mathrm{L}^{3} \cdot \mathrm{L}^{-1} \cdot \mathrm{T}^{-1}\right)$

qd : débit d'apport diffus $\left(\mathrm{L}^{3} \cdot \mathrm{L}^{-1} \cdot \mathrm{T}^{-1}\right)$

qp : débit $d^{\prime}$ apport ponctuel $\left(\mathrm{L}^{3} \cdot \mathrm{T}^{-1}\right)$

$\mathrm{R}_{\mathrm{H}}$ : rayon hydraulique $(\mathrm{L})$

$t$ : temps $(T)$

$\mathrm{U}$ : vitesse longitudinale moyenne ( $L . \mathrm{T}^{-1}$ )

$u_{i}$ : vecteur vitesse moyenne $\left(L . T^{-1}\right)$

$\mathrm{Up}^{*}$ : vitesse de frottement au ford $\left(\sqrt{\mathrm{g} \cdot \mathrm{I} \cdot \mathrm{R}_{\mathrm{H}}}\right)\left(\mathrm{L} \cdot \mathrm{T}^{-1}\right)$

$x$ : distance sur l'axe longituoinal (L)

$\alpha$ : pente des berges, fruit des berges

$\alpha_{i}$ : coefficient rentrant dans le calcul de BDL

$B D L$ : ccefficient rentrant dans le calcul de $D_{L}$

$\beta_{i}$ : coefficient rentrant dans le calcul de $\beta D L$

$\delta$ : fonction de Dirac

Tq : proportion du débit d'apport ponctuel par rapport à l'apport total

1 - INTRODUCTION

L'intérêt des hydrobiologistes pour les modèles écologiques est relativement récent mais depuis quelques années, la construction et 1'utilisation de modèles d'écosystèmes aquatičues se sont considérablement accélérées. Un grand nombre d'ouvrages a déjà été publié à ce sujet : PATTEN (1975), CANAIE (1976), HALL et DAY (1977), INNIS et O'NEIL (1979), JORGENSEN (1979), RINALDI et al., (1979), SCAVIA et ROBERTSON (1979), BIWAS (1981), DUBOIS (1981), MITSCH et al., (1981), BECK et Van STRATEN (1983), JORGENSEN (1983), JORGENSEN et MITSCH (1983), LAUENROTH et al., (1983), ORLOB (1983), BECK (1985), STRASKRABA et GNAUCK (1985), JORGENSEN (1986). 
La modélisation des écosystèmes permet de synthétiser et d'intégrer, à l'aide de différentes relations mathématiques, les connaissances acquises sur ces systèmes. Leurs simulations numériques en donnent une vision analytique et mécaniste. Elles permettent notamment, d'en obtenir des images dynamiques et de tester d'une part, la cohérence du savoir acquis, d'autre part, différentes hypothẽses sur leur structure et leur fonctionnement.

Les résultats exposés ici constituent l'aboutissement de la première phase d'un travail qui a pour objectif l'écriture d'un modèle écologique d'écosystẽme lotique. Cet outil doit permettre de mieux apprêhender les phénomènes d'auto-épuration grâce auxquels un cours d'eau retrouve un nouvel équilibre à la sui.te d'un enrichissement par un apport organique (WUHRMANN, 1972).

La principale caractéristique des écosystènes lotiques est un écoulement longitudinal dont la modélisation nécessite la prise en compte de l'hydrodynamique et des mécanismes de transport. Le txavail présenté ici est consacré exclusivement à cet aspect du modèle global.

2 - Milieu d'étude - Campagnes de mesures

L'expérimentation a été conduj.te sur l'Albenche (Savoie, France), cours d'eau de basse montagne (2ème ordre) situé dans le bassin versant du lac du Bourget (fig. 1). Ses principales caractéristiques sont les suivantes :

- bassin versant à caractère agricole, superficie $18 \mathrm{~km}^{2}$;

- longueur totale $11.6 \mathrm{~km}$; longueur étudiếe $3.2 \mathrm{~km}$;

- largeur moyenne $2.5 \mathrm{~m}$ (de $1 \mathrm{~m}$ à $4 \mathrm{~m}$ ) ;

- pente du cours d'eau dans son ensemble, de 1.2 à 2.5 ;

- pente moyenne du tronçon étudié $2 \%$;

- profondeur moyenne $0.2 \mathrm{~m}$ (de 0.05 à $0.6 \mathrm{~m}$ avec alternance de faciès a caractère lotique et lentique) ;

- valeurs extrểmes du débit instantané pendant la période d'étude : 15 à $60001 . \mathrm{s}^{-i}$;

- sinuosité non négligeable.

Les caractéristiques de largeur et de profondeur de 1'Albenche peuvent varier de façon considérable en un même point en fonction du régime hydrologique.

Ce ruisseau reçoit en anont de son cours, deux fois par jour, les eaux de lavage d'une porcherie, puis présente en aval tous les stades d'une auto-épuration menée pratiquement à son terme.

Le tronçon étudié s'étend sur $3.2 \mathrm{~km}$ à partir du point de rejet de la porcherie (station sup. : $430 \mathrm{~m}$; station inf. : $360 \mathrm{~m}$ d'altitude). 


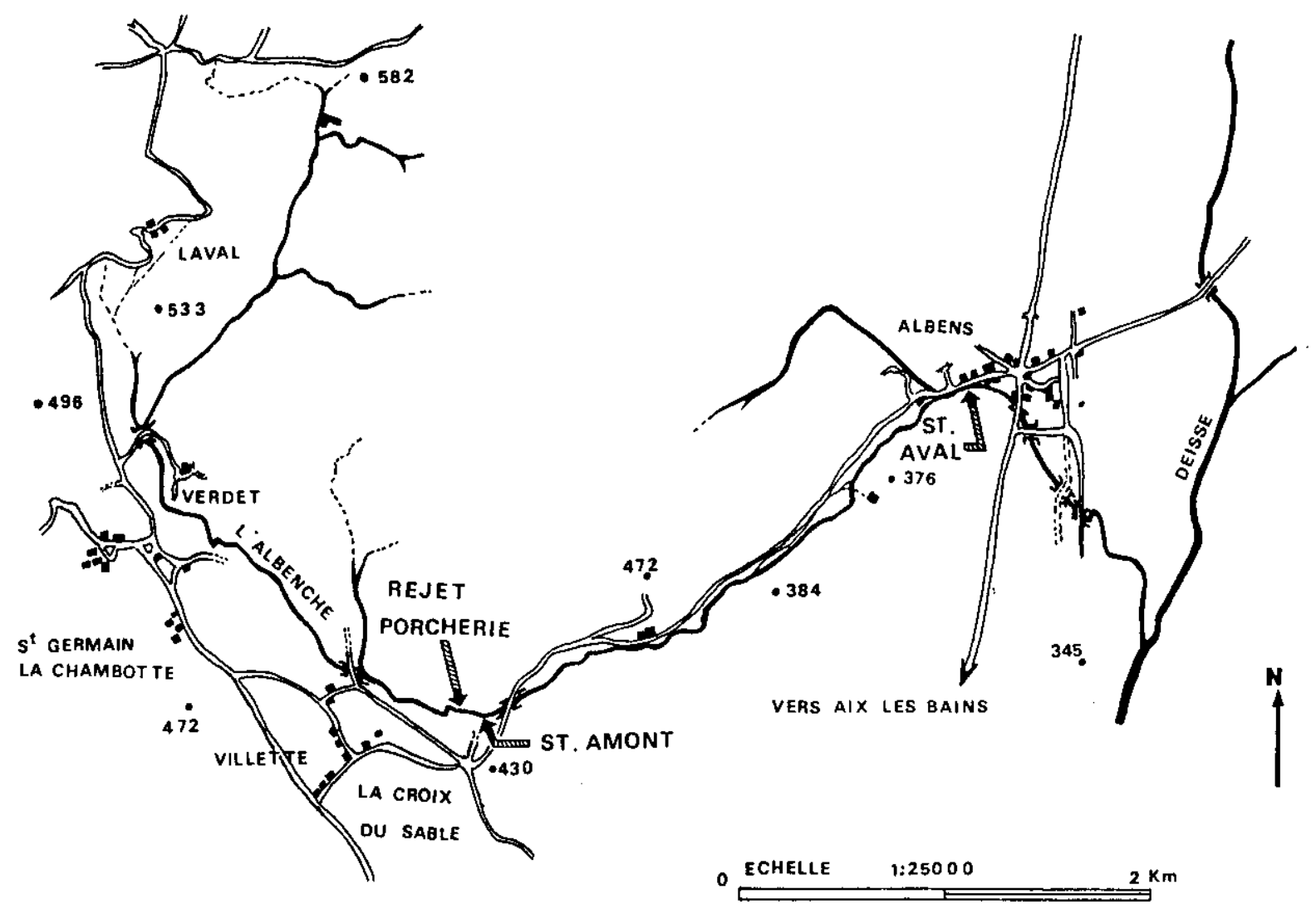

Figure 1.- L'Albenche.

Figure 1.- The Albenche river. 
Pour évaluer 1 'importance relative des processus participant à l'autoépuration, des mesures ont été effectuées pendant deux périodes :

- la première d'octobre 1981 à avril 1982 ;

- la seconde de novembre 1982 à mars 1984.

Les mesures réalisées lors de chaque période ont porté sur les principaux compartiments du système (fig. 2). Elles ont été effectuées selon les méthodes décrites dans les travaux précédemment publiés à ce sujet (FONTVIEILIJE et CAZELIES, 1985 ; FONTVIEILLE, 1987 ; FONTVIEILLE et CAZELLES, 1988). En ce qui concerne le compartiment aquatique, rappelons que chaque période est constituée de plusieurs campagnes de prélèvements ( 8 pour la première et 30 pour la seconde) de 27 heures chacun. Durant ces campagnes, 1 'eau est analysèe à raison d'un èchantillon par heure, chacun étant constitué par le mẻlange de quatre prélèvements effectués à un quart $d^{\prime}$ heure d'intervalle.

Les principaux résultats des travaux préliminaires (FONTVIEILLE et CAZELIES, 1985 ; FONTVIEILLE, 1987 ; FONTVIELLE et CAZELLES, 1988) ont mis en ếvidence :

- la faible importance des processus biologiques dans I'auto-épuxatior. durant les périodes de fortes eaux ;

- le rôle prépondérant des crues et donc des mécanismes hydrodynamiques,

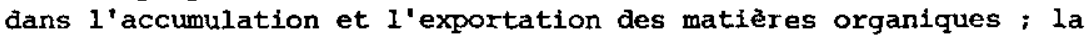
charge organique des sédiments de 1 'Albenche semble ettre conditionnée essentiellement par l'alternance de phases cumulatives et de phases émissives déterminées par le régime hydrologique.

\section{3 - Modélisation des fLUX DE CARBone oRganique}

La première campagne de mesures a permis d'appréhender les principales caractéristiques du systẻme et de son environnement. Son interprétation, completée par une recherche bibliographique (BOLING et al., 1975 ; DE ANGELIS et al., 1975 ; CLESCERI et al., 1977 ; Mac INTTRE et COLBY, 1978 ; O'NEIL et al., 1979 ; INEBSTER et al., 1979 ; THIEREN et al., 1981 ; NEWWBOLD et al., 1983 ; WEBSTER, 1983) a conduit à la structure du modèle schématisée à la figure 3 .

L'écriture du modèle est centrée sur les transferts de carbone organique et non sur ceux de l'oxygène en raison des particularités du cours d'eau :

- apports trophiques allochtones largement dominés pax le carbone organique :

- écculement fortement turbulent assurant une oxygénation telle que les deficits locaux ont pu être nẹgligés.

Dans les petits cours d'eau comme l'Albenche, le rôle du compartiment benthique a une importance prépondérante en ce qui concerne les processus détritiques (LOCK, 1981 ; HARREMOES, 1982 ; HVITVED-JACOBSEN, 1982 ; BOYLE et SCOTT, 1984 ; LOCK et al., 1984). 


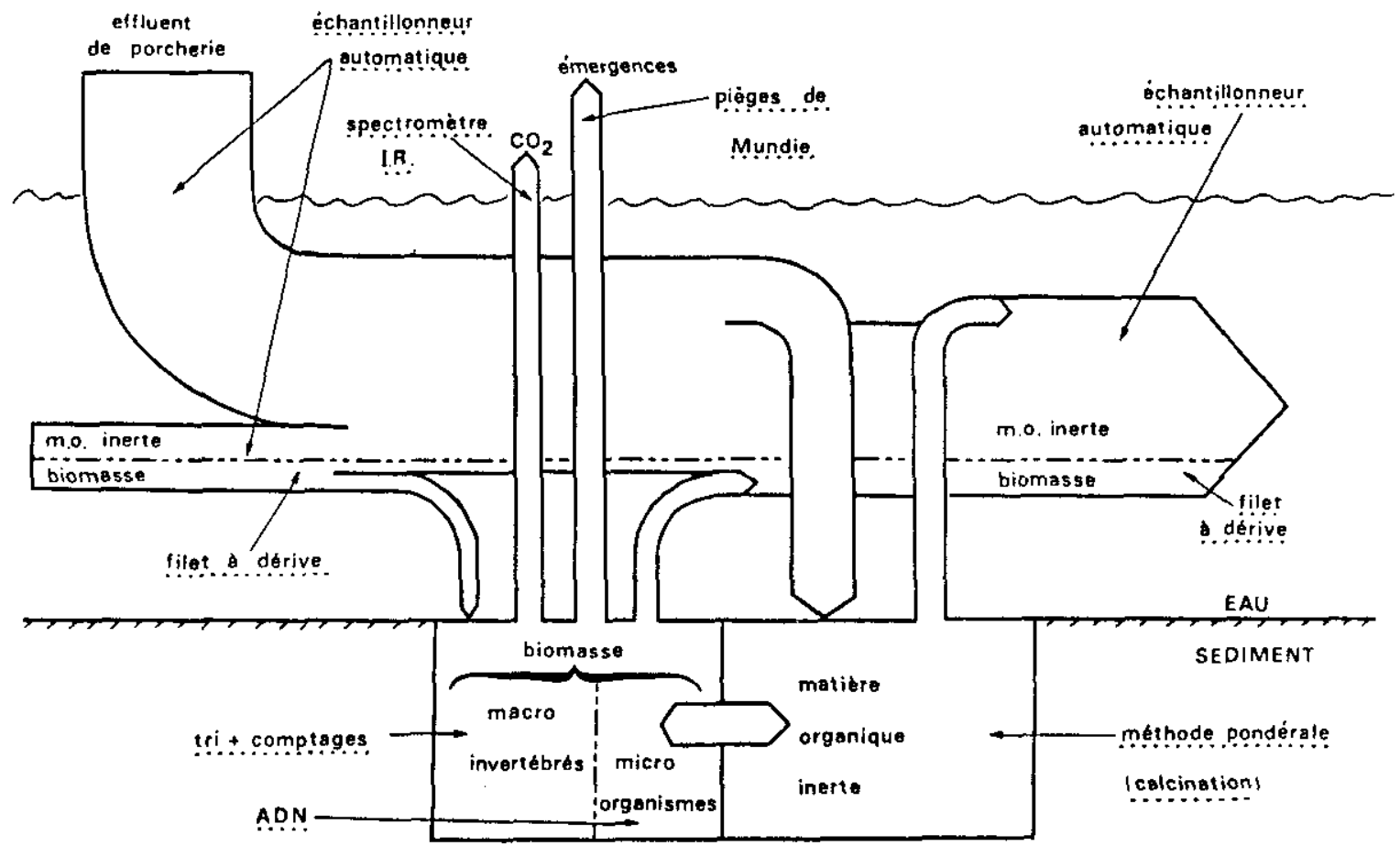

Figure 2.- Diagrame des compartiments carbonés mesurés dans l'Alberche.

Figure 2. - Schematic representation of the components of the carbon cycle taken into consideration in the Albenche river. 


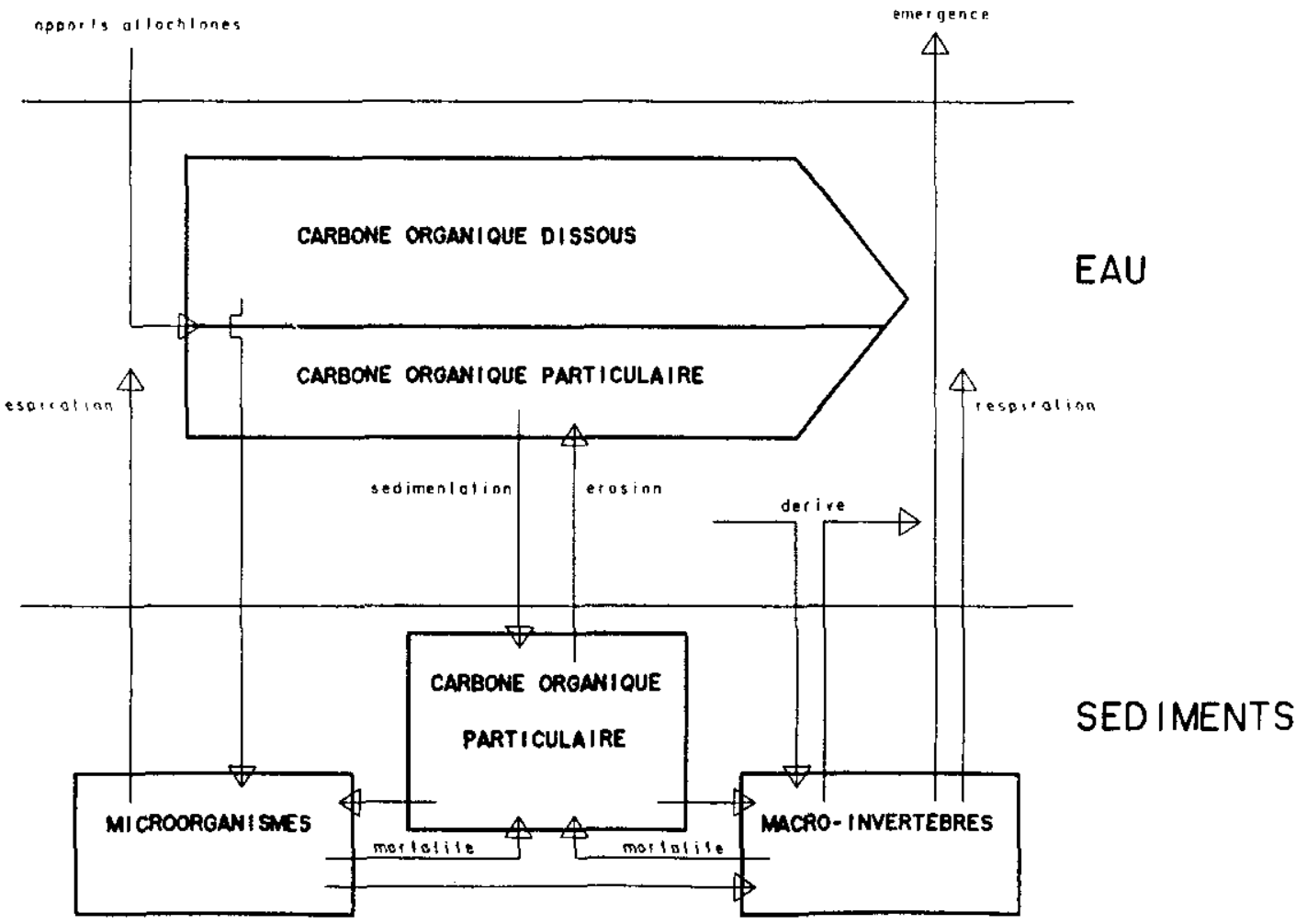

Figure 3.- Schématisation du modelle des flux de carbone organlque dans l'Albenche. Figure 3.- Schematic representation of the model simulating the carbon fluxes in the Albenche river. 
Les mesures effectuées sur l'Albenche montrent bien que les phénomènes biologiques sont négligeables à l'intérieur même de la masse d'eau, (inférieurs au seuil de détection de la méthode de mesure de la production de $\mathrm{CO}_{2}$ (FONTVIEILlE et RENAUD, 1982)). De ce fait seuls les phénomènes se déroulant dans le compartiment benthique sont. considérés.

Le modèle global décrit l'évolution de cinq variables d'état : le carbone organique dissous $\mathrm{C}$, le carbone organique particulaire en suspension $\mathrm{Cps}$, le carbone organique particulaire benthique $\mathrm{Cpb}$, la biomasse des microorganismes $B i$ et la biomasse des macroinvertébrés $\mathrm{Ba}$. Il permet de considérer éventuellement différentes classes de taille de carbone organique particulaire. Les transferts entre ces différents compartiments sont régis par des processus physiques (convection, diffusion, sédimentation ...) et/ou physiologiques (assimilation, excrétion, mortalité ....).

Basée sur le principe de conservation de masse, la formulation mathématique du modèle est constituée par un système d'équations aux dérivées partielles (1) qui décrivent l'évolution de chaque compartiment en fonction du temps et de la distance longitudinale, cette représentation spatio-temporelle permet de coupler les processus biologiques et les processus physiques, couplage essentiel à la description correcte de la dynaraique $d$ 'un tel écosystème.

$$
\begin{aligned}
& \frac{\partial C}{\partial t}=\text { transport }- \text { consommation par Bi }+ \text { apports } \\
& \frac{\partial C p s i}{\partial t}=\text { transport } i \text { - sédimentation } i+\text { érosion } i+\text { apports } i
\end{aligned}
$$

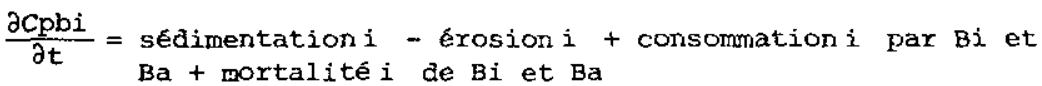

$$
\begin{aligned}
& \frac{\partial B i}{\partial t}=\underset{- \text { assimilation } . \text { consommation de } \mathrm{C} \text { et } \mathrm{Cpbi} \text { - respiration }}{ } \\
& \frac{\partial B a}{\partial t}=\text { assimilation.consonmation de } \mathrm{Cpbi} \text { et } \mathrm{Bi} \text { - respiration } \\
& \text { - mortalité } \pm \text { dêrive - émergence }
\end{aligned}
$$

La modélisation des mécanismes de trarsport pernet de décrire l'êvolution spatio-temporelle des concentrations en carbone organique et particulaire dans la veine liquide et dans les sédiments. Les concentrations prédites permettent de décrire l'êvolution des compartiments biologiques, puis d'interpréter le role de ces compartiments dans le fonctionnement global du système (CAZELLES, 1987).

Seule la modélisation des termes liês aux mécanismes de transport du matériel organique dissous sera développée ici. Le détail de la construction du modêle global (1) et de son application, ont été décrits dans un précédent travail (CAzELLES, 1987) et fera l'objet de publications ultérieures. 


\section{4 - Modélisation de L'hydrodynamique et des mécanismes DE TRANSPORT}

Le melange et le transport de substances solubles dans l'écoulement d'un cours d'eau sont des phénomènes complexes. Ils résultent d'interactions entre les mécanismes de convection et de turbulence (SAYRE, 1973; FISCHER, 1973). Ces deux mécanismes sont fortement dépendarts des caractéristiques de l'écoulement (champ de vitesse, frottements, etc. et de la géométrie de l'écculement).

La modélisation du transport des substances solubles dans l'Albenche a été réalisée à partir de deux modèles : un modèle hydrodynamique et un modèle de transport. Le modèle hydrodynamique permet d'accéder aux caractéristiques de l'écoulement nécessaires à la mise en oeuvre du modêle de transport.

L'Albenche est un petit cours d'eau à forte pente où alternent les zones lentes et les zones rapides. Dans ce type d'eau courante, le caractère non-uniforme de $1^{\prime}$ écoulement est accentué par les apports latéraux et les êchanges avec le sous-écotilement (HUFF et al., 1982 ; BENCALA et al., 1983 ; BENCALA et WALTERS, 1983). Cette non-uniformité a été parfaitement vérifiée expérimentalement dans le cas de l'Albenche.

Le rơle prépondérant des crues dans le fonctionnement de l'écosystème (FONTVIEILLE et CAZELIES, 1985) a, en outre, justifié I'introduction dans les modèles de termes permettant la description de périodes de rêgimes non-stationnaires.

L'ensemble de ces contraintes nous a obligés à construire des modẻles unidirectionnels, non uniformes et non stationnaires.

\section{4-1 Modèle hydrodynamique}

Les contraintes exposées ci-dessus nous ont conduits à utiliser dans un premier temps, le modèle classique de Saint-Venant en considérant que :

- les calculs hydrodynamiques ne sont pas affectés par la composition de 1 'eau :

- le mêllange est complet dans chaque section de l'écoulement :

- la forme géométrique des sections est trapézoldale.

$$
\begin{aligned}
& \frac{\partial A(x, t)}{\partial t}+\frac{\partial Q(x, t)}{\partial x}=q(t) \\
& \frac{\partial u(x, t) \cdot A(x, t)}{\partial t}+\frac{\partial U^{2}(x, t) \cdot A(x, t)}{\partial x}+g \cdot A(x, t) \cdot \frac{\partial H(x, t)}{\partial x} \\
& =g \cdot(I(x)-J(x, t)) \cdot A(x, t)-v(x, t) \cdot q(t)
\end{aligned}
$$




$$
\begin{aligned}
& A(x, t)=H(x, t) \cdot\left(B F+\frac{2 \cdot H(x, t)}{t g \alpha}\right) \\
& B(x, t)=B E+2 \cdot H(x, t) / \operatorname{tg} \alpha \\
& P m(x, t)=B F+2 \cdot H(x, t) / \sin \alpha \\
& R_{H}(x, t)=A(x, t) / P m(x, t) \\
& q(t)=\frac{C q \cdot Q(O, t) e q}{d} \\
& J(x, t)=\frac{U(x, t)^{2}}{k^{2} \cdot R_{H}(x, t)^{4 / 3}}
\end{aligned}
$$

A partir des valeurs expérimentales de $A(x, t), H(x, t), B(x, t)$ mesurées au niveau des stations amont et aval, un algorithme d'optimisation nous a pexmis d'obtenir les valeurs optimum de la largeux au radier (BF) et du fruit des berges $(\alpha): B F=1.5 \mathrm{~m} ; \alpha=17^{\circ}$.

Dans un cours d'eau comme l'Albenche, les frottements ont une grande importance et le calcul des pertes de charges $(J(x, t)$ par une relation semi-empirique comme celle de STRICKIER (5) $n^{\prime}$ est pas satisfaisante (BEDFORD et $a l$., 1983). De plus, les caractéristiques de l'écoulement sont extrêmement hêtéxogênes, aussi bjen dans 1'espace (alternances de zones à faciès lotique et à faciès lentique), que dans le temps. Les crues peuvent modifier la géométrie du cours d'eau au point que pour un même débit, les caractéxistiques hydrodynamiques d'une mêne station (surface mouiliée, coefficient de frottement ...) soient totalement modifiées. Dans ce cas, le coefficient de frottement $k$ ne peut plus etre considéré comme constant et son estimation pose de nombreux problèmes (JARRETT, 1984).

Compte tenu des vaxiations des caractéristiques de l'écoulement, nous avons opté pour une simplification du modèle : l'equation de continuité du modẻle de Saint-venant a été conservěe et l'équation dynamique remplacé par une equation empirique (IEOPOLD et $a l_{*}$, 1964) utilisée par de nombreux hydrobiologistes :

$$
\begin{aligned}
& \frac{\partial A(x, t)}{\partial t}+\frac{\partial Q(x, t)}{\partial x}=q(t) \\
& A(x, t)=c a \cdot Q(x, t) e^{e a}
\end{aligned}
$$

Dans le tableau 1 sont portés différents résultats du modèle hydrodynamique. Ce tableau est une bonne illustration de la grande variabilité des caractéristiques de l'écoulement de l'Albenche. 


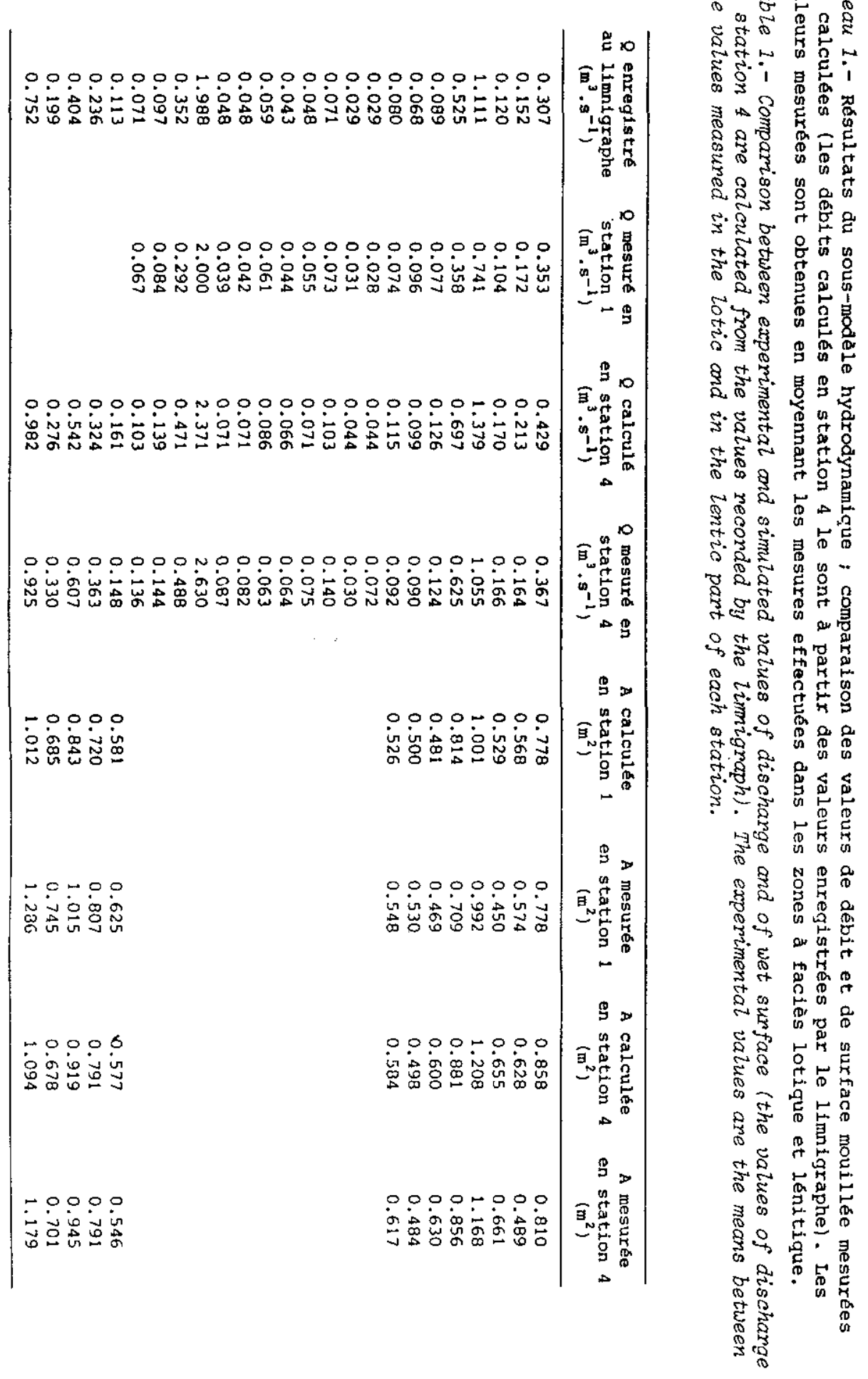




\section{4-2 Modè1e de transport}

Toutes les représentations mathématiques des phénomènes de transport et de mélange sont basées sur le principe de conservation d'une grandeur scalaire transportée par l'écoulement. Elles sont fondées sur les équations de bilan des eaux de surface qui expriment 1'évolution des paramè-

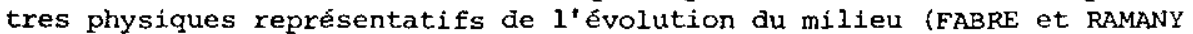
BALA, 1980 ; CAUSSADE et al., 1982). A partir de ces équations de bilan des eaux de surface, on peut écrire des nodèles pratiques applicables à des données expérimentales de terrain, à l'aide d'hypothèses supplêmentaires :

- simplification à l'aide de l'"approximation" de Boussinesg, qui permet, notamment, de ne pas tenir compte des effets des variations de masse volumique ;

- prise en compte des moments statistiques des variables locales instantanées pour un écoulement turbulent.

On aboutit, ainsi, à l'équation classique de convection-diffusion pour un écoulement tridimensionnel :

$$
\frac{\partial c}{\partial t}+u_{i} \cdot \frac{\partial c}{\partial x_{i}}=\frac{\partial}{\partial x_{i}}\left(\operatorname{rxi} \cdot \frac{\partial c}{\partial x_{i}}\right)
$$

avec i directions de 1 'écoulement.

Dans le cas d'un cours d'eau comme l'Albenche, l'écoulement. se fait préférentiellement dans la direction longitudinale. On peut donc intégrex l'équation (7) sur une section droite de l'écoulement, de façon à obtenir une équation unidimensionnelle (nodèle unifilaire) exprimant les variations des variables uniquement dans le sens de l'écoulement. On obtient alors :

$$
\frac{\partial A(x, t) \cdot C(x, t)}{\partial t}+\frac{\partial Q(x, t) \cdot C(x, t)}{\partial x}=+\frac{\partial}{\partial x}\left(A(x, t) \cdot D_{L}(x, t) \cdot \frac{\partial C(x, t)}{\partial x}\right)
$$

Dans cette equation, $D_{L}$ est le coefficient de dispersion longitudinale qui exprime l'influence des profils de vitesse et de la répartition des concentrations dans les sections mouillêes de 1 'écoulement. De nombreuses formulations ont été prcposées pour le calculer, soit à partir des caractéristiques hyarodynamiques de l'écolilement (ELDER, 1959 ; FISCHER, 1967, 1968 ; LIU, 1977 ; LIU et CHENG, 1980), soit à partir d'expériences de traçages (FISCHER, 1964). Dans notre cas, la formulation adoptée est celle de LIU (1977) :

$$
\begin{aligned}
& D_{L}(x, t)=B D L \cdot \frac{U(x, t)^{2} \cdot B(x, t)^{3}}{U p^{*}(x, t) \cdot A(x, t)} \\
& B D L=\alpha_{i} \cdot\left(\frac{U(x, t)}{U p_{*}(x, t)}\right)^{\beta_{i}}
\end{aligned}
$$

avec i numéro du troņ̧on étudié lorsque plusieurs tronçons sont considêrếs.

Pour appliquer I'équation (8) au cas d'une substance non-conservative, differents termes, sources et puits, sont introduits. L'équation (10) permet de prendre en compte des apports diffus $(q(t) . \mathrm{Cq}(x, t))$ et une disparition (Kd. $A(x, t) \cdot C(x, t))$ : 


$$
\begin{aligned}
& \frac{\partial A(x, t) \cdot C(x, t)}{\partial t}+\frac{\partial Q(x, t) \cdot C(x, t)}{\partial x}=C q(t) \cdot q(t)-K d \cdot A(x, t) \cdot C(x, t) \\
& +\frac{\partial}{\partial x}\left(A(x, t) \cdot D_{L}(x, t) \cdot \frac{\partial C(x, t)}{\partial x}\right)
\end{aligned}
$$

\section{4-3 Ajustement des paramètres des modèles}

Les deux équations du modèle ( 6 et 8 ) sont résolues par des méthodes aux difféxences finies utilisant des schémas de discrétisation implicite..

Les paramètres sont ajustés à l'aide :

- de valeurs expérimentales de débits et de surfaces mouillées mesurées au niveau des stations amont et aval (fig. 1) ;

- d'expériences de traçage à l'aide d'un traceur fluorescent, la rhodamine $\mathrm{B}$.

En ce qui concerne l'équation (9), dans un premier temps BDL est considéré comme un paramètre à ajuster lors de chaque simulation.

Certaines caractéristiques hydrodynamiques de l'écoulernent, lors de chaque campagne de traçage simulếe, sont portées dans le tableau 2.

Tableau 2.- Valeurs (pour un régime quasi stationnaire) et plages de valeurs (pour un régime non-stationnaire) de certaines caractéristiques hydrodynamiques de 1 'Albenche durant les experiences de traçage.

Table 2.- Hydrodynamic characteristics of the Albenche river during the dye tracing experiments.

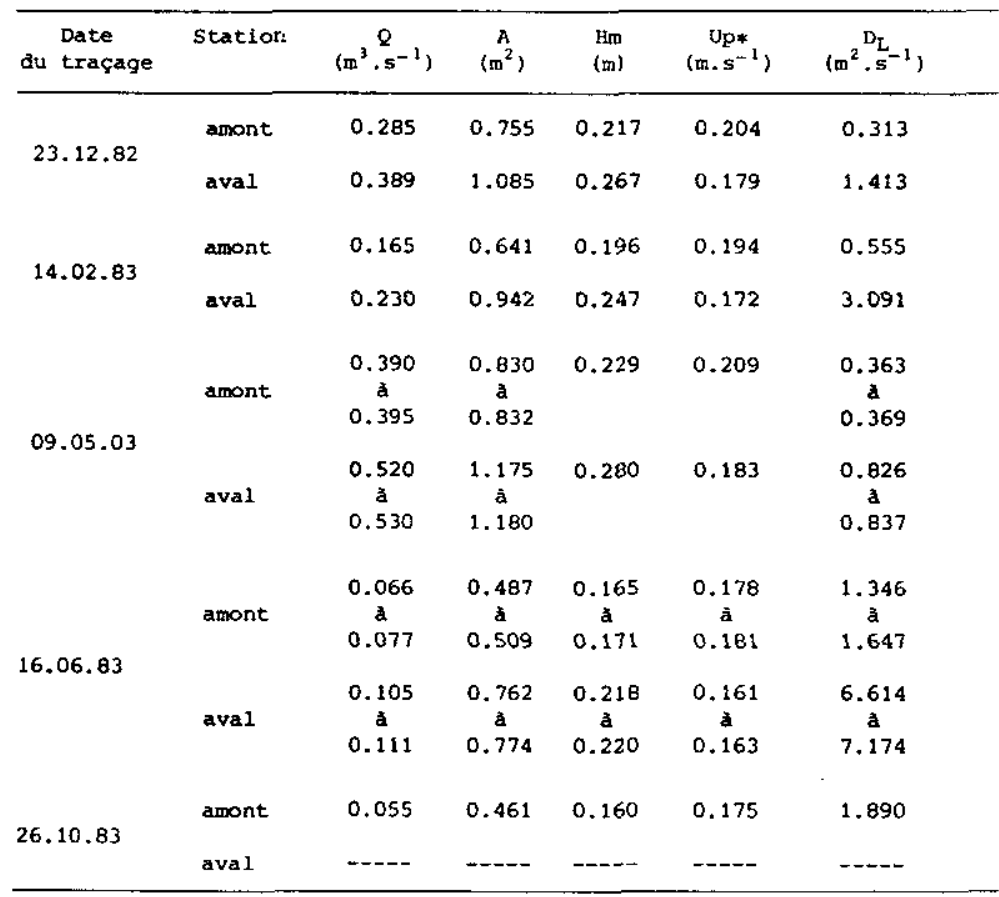


Une analyse exhaustive du comportement des deux sous-modèles aux variations de chaque paramètre a été effectuée (CAZELLES, 1987). Elle a permis de souligner l'importance des paramètres liés à la convection et donc de 1 'hydrodynamique pour positionner correctement le pic des nuages simulés par rapport au pic des nuages expérimentaur, et des paramètres liés à la dispersion longitudinale pour ajuster la forme des nuages simulés.

La sensibilité du modèle de transport aux caractéristiques hydrodynamiques du ruisseau s'est révélée telle qu'il a fallu prendre en compte une rupture de pente au point $2400 \mathrm{~m}$ et $\mathrm{I}^{\prime}$ apport ponctuel d'un petit affluent au point $2900 \mathrm{~m}$.

En conséquence, deux lots de paramètres ont été constitués (correspondant respectivement à I'anont et à $I^{\prime}$ aval du point $2400 \mathrm{~m}$ ). En outre, le débit d'apport a été subdivisê en un débit d'apport ponctuel et un débit d'apport alffus réparti sur l'ensemble du tronçon étuđié. Pour tenir compte de ces éléments, les éqquatiors (4) et (6) sont remplacées par les équatiors (11) et (12) :

$$
\begin{aligned}
& \left.q u(t)=\left(\frac{1-T q}{d}\right) \cdot c q \cdot Q(0, t) e q\right] \\
& q p(t)=T q \cdot C q \cdot Q(0, t) e q \\
& \frac{\partial A(x, t)}{\partial t}+\frac{\partial Q(x, t)}{\partial x}=q d(t) \\
& Q(x, t)=Q(x, t)+\delta(x-2900) \cdot q P(t) \\
& A(x, t)=c a i \cdot Q(x, t)
\end{aligned}
$$

La figure 4 illustre la senstbilité du modèle aux caractéristiques hydrodynamiques de l'écoulement. I'ajustement des valeurs calculées aux valeurs expérimentales dans la station aval nécessitait l'affectation de valeurs, qui nous ont semblēes non réalistes, au coefficient $B D L$ de 1 'équation (9). Ces valeurs étaient trois à dix fois plus élevés que celle de la station amont (cf. legende fig. 5). Un terme de disparition de la rhodamine a donc eté introduit dans l'equation de transport (fig. 5) en correspondance avec les phénomènes d'adsorption et de photodégradation constatés par différents auteurs (BENCAIA et al., 1983 ; BUJON, 1983).

Les résultats de la simulation des expériences de traçage à des débits différents sont portés sur la figure 6. Elle montre que les modèles utilisés ainsi que la valeur des paramètres adoptés permettent de retrouver l'allure des variations et les ordres de grandeur des concentrations. Ces résultats peuvent être considérés come satisfaisants en regard :

- des problèmes posés par la grande variabilité des caractéristiques de 1'écoulement et par sa complexité (JARRETT, 1984) ;

- de l'adoption d'hypothèses simplificatrices dans l'établissement des modeles ; il faut souligner que le modèle simule des concentrations moyennes dans l'espace, alors que les valeurs expérimentales ne sont que des valeurs ponctuelles mesurées dans la zone la plus représentative de l'écoulement de chaque station. 

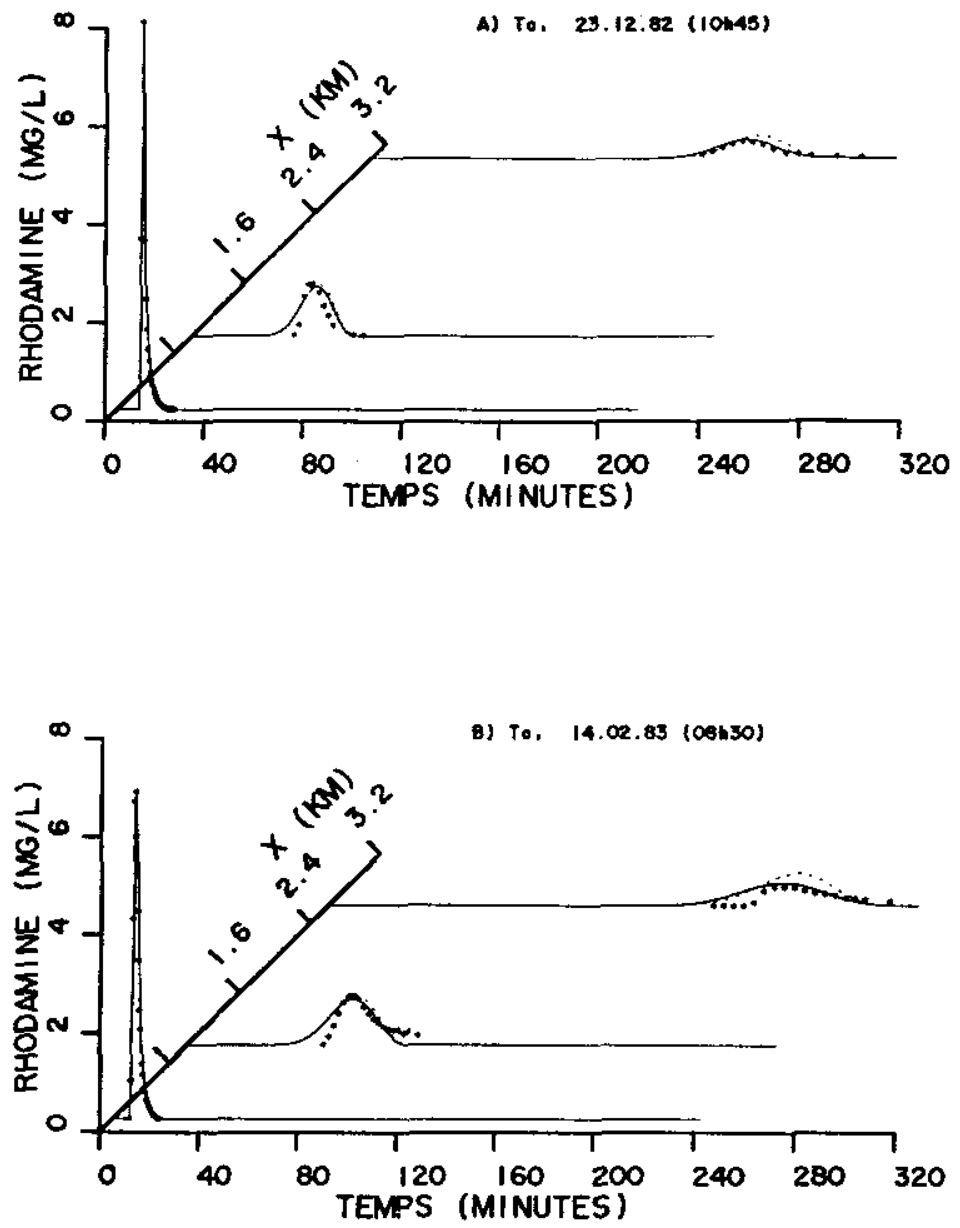

Figure 4.- Sensibilité du modèle aux caractêristiques hydrodynamiques du cours d'eau.

Figure 4.- Sensibility $0_{j}^{*}$ the model in relation to the hydrodynamic characteristics of the stream.

[a : $Q=285 \mathrm{l} / \mathrm{s} ; \mathrm{b}: Q=165 \mathrm{l} / \mathrm{s} ; *$ : valeurs expérimentales]

- ligne pointillée : prise en compte d'un seul tronçon et d'un seul lat de paramètres $[a: \beta=0.008, T q=0.00 ; b: \beta=0.03, T q=0.00$ ) ;

- ligne continue : príse en compte de 2 tronçons et donc de 2 lots de paramètres ainsi que du petit affluent au kilomètre 2.9

(a $: \beta(1)=0.008, \beta(2)=0.15, \mathrm{Tq}=0.2 ; b: \beta[1]=0.03, \beta(2)=0.5$, $\mathrm{T} Q=0.2\}$.

( $a: Q=285 \mathrm{l}: \mathrm{s} ; \mathrm{Q}: Q=165^{\circ} \mathrm{l} / \mathrm{s} ; *$ : experimental values $]$

- brocken line : considering the river as a single stretch, with a single set of parameters $\{a: \beta=0.008$, Tq $=0.00, B=0.03$, Tq $=0.00\}$;

- solid line : considering :

1) two stretches in the river and thus two sets of parameters and

2) the small tributary at the point $2.9 \mathrm{~km}(a: B(1)=0.008$,

$B(1)=0.008, B(2)=0.15, T q=0.2 ; b: \beta(1)=0.03, B(2)=0.5 x$ $T_{q}=0.21$. 

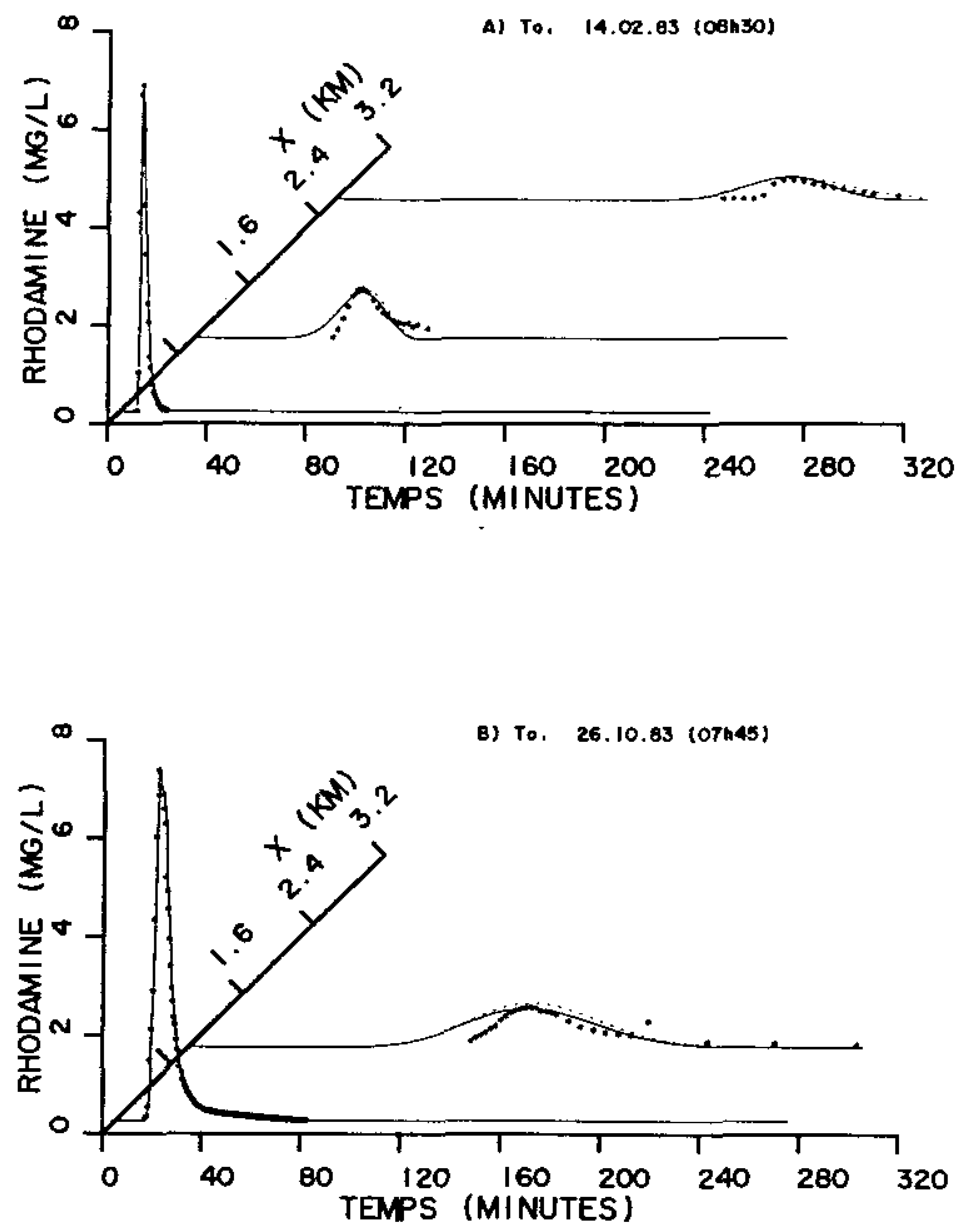

Figure 5.- Influence de l'introduction d'un terme de disparition sur le comportement des modèles lors de simulation de campagnes de traçage.

Figure 5.- Consequences of the introduction of a term which simulates the disappearence of the dye on the simulation of the dye tracer experiments.

(a: $Q=165 \mathrm{l} / \mathrm{s} ; B=Q 55 \mathrm{l} / \mathrm{s} ; *$ : valeurs expérimentales)

- ligne pointillée : $(a: B(1)=0.03, B(2)=0.50, \mathrm{Kd}=0$; $b: B(1)=0.45, B(2)=1, K d=0)$;

- Iigne continue : prise en compte d'un terme de disparition $\left(a: B(1)=0.03, B(2)=0.15, K d=1.510^{-5} ; b: \beta(1)=0.45\right.$, $B(2)=1, K d=1.5\left(0^{-5}\right)$.

(a: $Q=165 \mathrm{l} / \mathrm{s} ; \mathrm{b}: Q=55 \mathrm{l} / \mathrm{s} ; *$ : experimental values)

- brocken line: $(a: B(1)=0.03, \beta(2)=0.50, K d=0 ; b=\beta(1)=0.45$. $B(2)=1, K d=0)$;

- solid line : taking in account the sink term : $[\mathrm{a}: B(1)=0.03$, $B(2)=0.15 ; \mathrm{Kd}=1.510^{-5} ; b: B(1)=0.45, \beta(2)=1, \mathrm{Kd}=510^{-5} \mathrm{\}}$. 

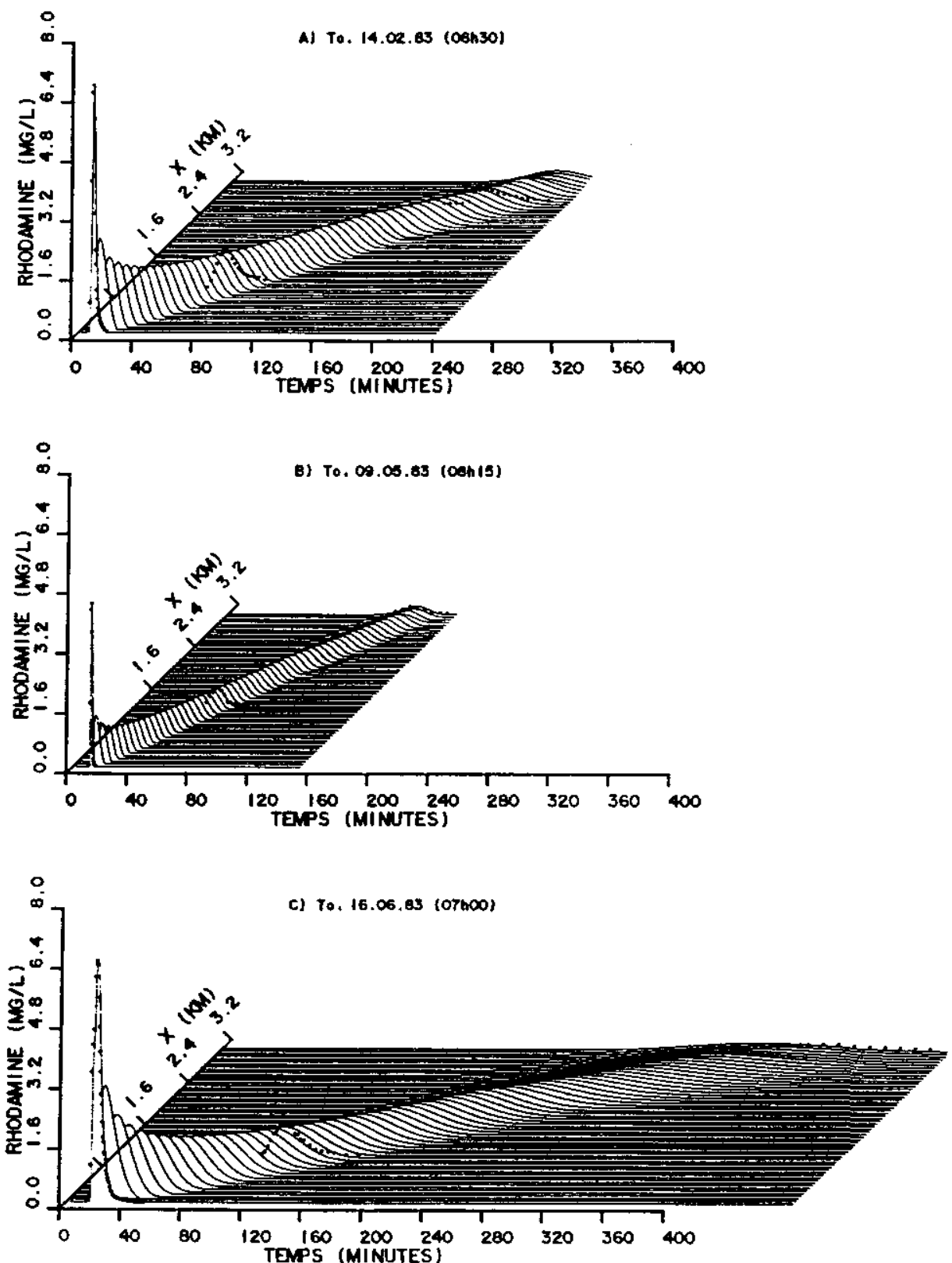

Figure 6.- Simulation d'expériences de traçage dans I'Albenche. Figure 6.- Simulation of dye tracer experiments in the Albenche river. (a: $Q=165 \mathrm{l} / \mathrm{s} ; \mathrm{b}: Q=395 \mathrm{l} / \mathrm{s} ; c: Q=77 \mathrm{l} / \mathrm{s} ; *$ : valeurs expérimentales\}.

$(a: Q=165 \mathrm{l} / \mathrm{s} ; \mathrm{b}: Q=395 \mathrm{l} / \mathrm{s} ; \mathrm{c}: Q=77 \mathrm{l} / \mathrm{s} ; *$ : experimental values\}. 
Dans le cas de faibles débits (fig. 6c) les valeurs calculées s'éloignent des valeurs expérimentales, le nuage simulé arrivant trop tardivement dans la station aval. Compte tenu de l'importance de ces périodes dans I'hydrologie de I'Albenche, il sera sans doute souhaitable pour la suite du travail, de prendre en compte d'autres phénomènes de mélange dans la structure du modèle de transport. Le stockage temporaire đes substances dissoutes dans les zones mortes dues aux irrégularités de I'écoulement et à son absence d'uniformité, semble en particulier d'un grand intérêt pour un petit cours d'eau comme l'Albenche (VALENTINE et WOOD, 1977 ; SABOL et NORDIN, 1978 ; BENCALA et WALTER, 1983).

\section{5 - ApPlication des modéles au tRansport du C.O.D. DANS L'ALBENCHE}

L'utilisation de ces modèles pour simuler le transport du carbone organique dissous (C.O.D.) dans 1 'Albenche, nécessitait la connaissance des valeurs des concentrations à l'entrée du tronçon étudié. Ces valeurs peuvent être rattachées a 2 catégories : celles observées dans le milieu en l'absence de rejets (le "bruit de fond" du ruisseau) et celles qui. concernent le rejet de la porcherie. Nous avons utilisé pour valeurs "d'entrée" les valeurs expérimentales de c.o.D. , mesurées au niveau de la station amont entre :

- le 15.08 .83 a 22 heures et le 17.08 .93 à 1 heure;

- le 04.10 .83 à 18 heures et le 05.10 .83 à 21 heures.

Les modèles simulent le transport du C.O.D. sur une période de 48 heures, avec un pas de temps de 15 minutes et sous 1 'hypothèse que le cycle de 24 heures des flux, mesuré au niveau de la station amont, se reproduise de façon identique pendant toute la période simulée.

Ces campagnes correspondent à des périodes d'étiage de l'Albenche durant lesquelles les variations de débit sont faibles. Ces dernières ont, néanmoins, été prises en compte lors des simulations.

Durant ces périodes d'étiage, l'apport du rejet de la porcherie est en moyenne de $150 \mathrm{~kg}$ de carbone par jour. II représente de 8 a 44 o de la charge naturelle de l'eau en carbone organique total de l'eau. Ia valeur du rapport entre le carbone dissous et le carbone particulaire du rejet varie de 10 à 160 (FONTVIEILIE, 1987).

Après avoir initialise les concentrations tout le long du cours d'eau, les modèles simulent le régime transitoire. Pour ces calculs, le C.O.D. est considéré comne une substance conservative. Une valeur nulle est donc affectée au terme de disparition dans le modele de transport ( $\mathrm{kd}=0$, équation (10). La valeur du "bruit de fond" du ruisseau en carbone organique dans la station amont est affectée à la concentration des apports (Cq) .

Les valeurs des paramètres sont celles obtenues aprảs ajustement grăce aux simulatiors du transport de la rhodamine. Ces valeurs, ainsi que celles de certaines caractéristiques de 1 'Albenche se trouvent dans les tableaux 3 et 4 . L'évaluation du coefficient BDL, n'était pas possible en 
raison du fait que les expériences de traçage $n$ 'ont pas été concomitantes avec les campagnes de mesures au C.O.D.. Il a été déterminé à l'aide de la deuxième relation de (9) et des valeurs obtenues a partir des traçages à la rhodamine (fig.7).

Tableau 3.- Caractéristiques de 1'Albenche lors des deux simulations du transport du C.O.D.. Ia concentration des apports (Cq) est considérée égale à celle du "bruit de fond" du cours d'eau, mesurée en 1 'absence de rejets.

Tabie 3.- Some characteristics of the Albenche miver used by the model for the D.O.C. transport simulations. The D.O.C. input (Cq) is taken equal to the background concentration of $D . O$.C. during the periods of absence of pigstry waste inflow.

\begin{tabular}{|c|c|}
\hline DATE & 15.08 .83 \\
\hline$\Omega$ & $\begin{array}{l}\text { station amont de } 21 \text { à } 291 . \mathrm{s}^{-1} \\
\text { station aval. de } 32 \text { à } 441 . \mathrm{s}^{-1}\end{array}$ \\
\hline A & $\begin{array}{l}\text { station amont de } 0.345 \text { a } 0.380 \mathrm{~m}^{2} \\
\text { station aval de } 0.592 \text { a } 0.641 \mathrm{~m}^{2}\end{array}$ \\
\hline Temperature & 14 à $17^{\circ} \mathrm{C}$ \\
\hline $\mathrm{Cq}$ & 120 à $250 \mathrm{mg} \mathrm{C.1^{-I }}$ \\
\hline $\mathbf{Q}$ & $\begin{array}{l}\text { station amont de } 29 \text { à } 38 l . s^{-1} \\
\text { station aval de } 44 \text { à } 571 . \mathrm{s}^{-1}\end{array}$ \\
\hline $\mathbf{A}$ & $\begin{array}{l}\text { station amont de } 0.380 \text { a } 0.412 \mathrm{~m}^{2} \\
\text { station aval de } 0.641 \text { a } 0.684 \mathrm{~m}^{2}\end{array}$ \\
\hline Température & 9.5 à $11.5{ }^{\circ} \mathrm{C}$ \\
\hline $\mathrm{Cq}$ & 250 à $340 \mathrm{mg} \mathrm{C.1}-:$ \\
\hline
\end{tabular}

Sur les figures 8 et 9, représentent les résultats des simulations du transport du C.O.D. a des dates différentes, on visualise les deux rejets quotidiens de la porcherie. On peut remarquer le fort étalement des rejets entre la station amont et la station aval, malgré leur faible dilution lorsque le débit est peu important $\left(21 \mathrm{l.s} \mathrm{s}^{-1}\right.$ le $15.08 \mathrm{et}$ $381 . \mathrm{s}^{-1}$ le 4.10 ). Ce fort etalement est due essentiellement a 1 'importance de la dispersion longitudinale. Lors de ces simulations le coefficient varie de 2 a $10 \mathrm{~m}^{2} \cdot \mathrm{s}^{-1}$, ce cui est relativement important pour un petit cours d'eau. Pour des cours d'eau du meme type, BENCALA et WALTER (1983) utflisent des coefficients de dispersion qui varient entre 0.1 et $0.5 \mathrm{~m}^{2} \cdot \mathrm{s}^{-}$at BAJRAKTAREVIC-DOBRAN (1982) des coefficients variant entre 0.1 et $4 \mathrm{~m}^{2} . \mathrm{s}^{-1}$. Sur ces figures, $1^{\prime}$ importance de la rupture de pente au point $2400 \mathrm{~m}$ sur l'etalement des nuages apparaft aussi, nettement. 
Tableau 4. - Valeurs des paramètres des modèles utilisées lors des simulations du transport du C.O.D.

Table 4.- Values of parconeters used by the model during the D.O.C. tronsport simulations

\begin{tabular}{|c|c|c|}
\hline PARAMETRES & $\begin{array}{l}\text { VALEURS } \\
\text { UTILISEES }\end{array}$ & SIGNIFICATION \\
\hline $\mathrm{cq}$ & 1.289 & - paramètres du calcul de la relation \\
\hline eq & 0.955 & entre le débit et le débit d'apport \\
\hline $\mathrm{ca}_{1}$ & 1.100 & - paramètres de la relation entre 8 et \\
\hline ea & 0.300 & A pour le premier et le deuxième \\
\hline $\mathrm{Ca}_{2}$ & 1.400 & txonçon \\
\hline ea 2 & 0.250 & \\
\hline$\alpha_{1}$ & 0.153 & - paramètres de la relation permettant \\
\hline$\beta_{1}$ & -3.607 & le calcul du coefficient de dispersion \\
\hline$\alpha_{2}$ & 0.492 & longitudinale (Dr) pour le premier \\
\hline$\beta_{2}$ & -3.768 & et le deuxième tronçon \\
\hline $\mathrm{Tq}$ & 0.200 & - proportion de l'apport ponctuel \\
\hline
\end{tabular}

Ces simulations montrent bien que, même si la distribution du C.O.D tout au long du cours d'eau, peut etre expliquée partiellement par les phénomènes hydrophysiques, ils sont insuffisants pour expliquer totalement les valeurs expérimentales mesurées. La surestimation des valeurs prédites par rapport aux valeurs mesurées, au niveau de la station aval, peut témoignex :

- du rôle, sur la disparition du C.O.D.; de phénomènes physiques ou chimiques non pris en compte dans le modẽle sous sa forme actuelle;

- et surtout, d'une consommation due à l'activité des biocoenoses benthiques.

\section{6 - CONCLUSIONS}

Le modèle global prcposé ((1) et fig. 3) fait la synthèse des deux types d'approche que $1^{\prime}$ on rencontre dans le domaine de la modelisation de systèmes aquatiques (SOMLYODY, 1982) :

- une approche hydraulique où l'aspect hydrodynamique est très développé et où, par contre, l'aspect biologique et biochimique est très réduit ; $c$ 'est le cas de beaucoup de modèle dit de qualitế d'eau ;

- une approche écologique où contrairement à la précédente, l'aspect hydraulique est peu ou pas développé (modèle ponctuel ou chémostat par exemple) mais où un certain réalisme écologique est introduit dans le modèle. 
a) TRONCON AMONT

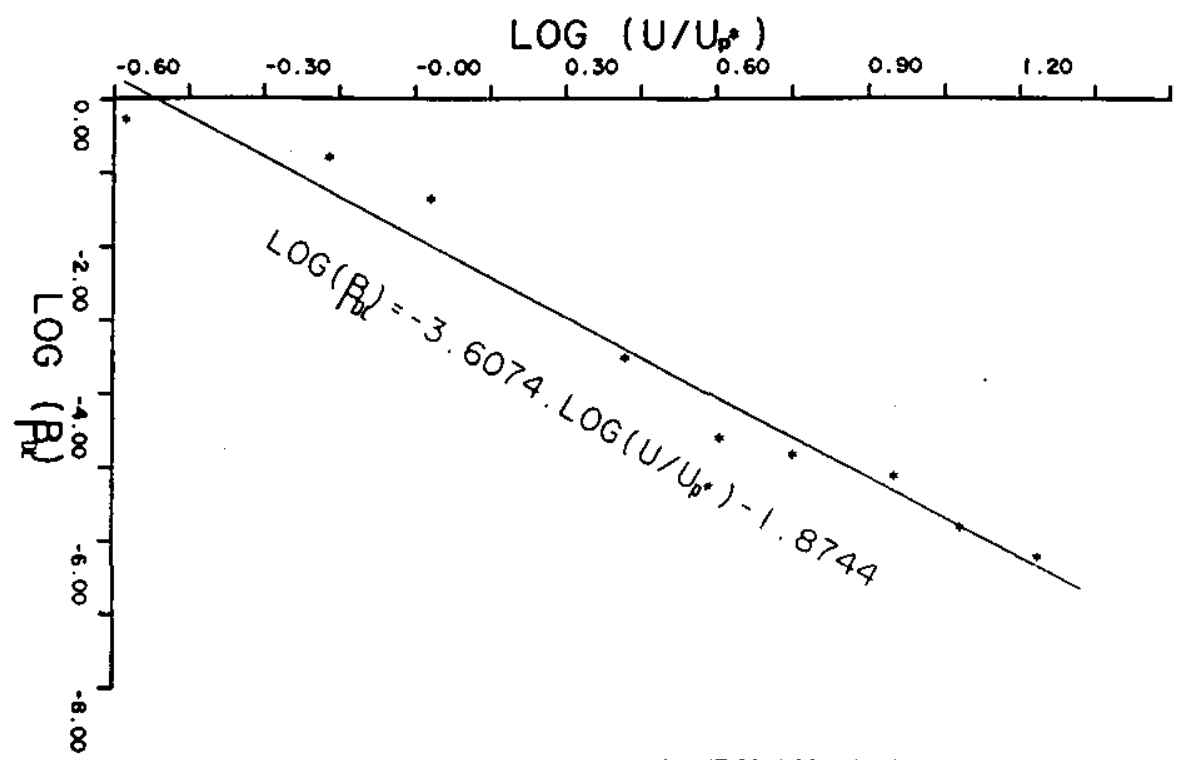

b) TRONCON AVAL

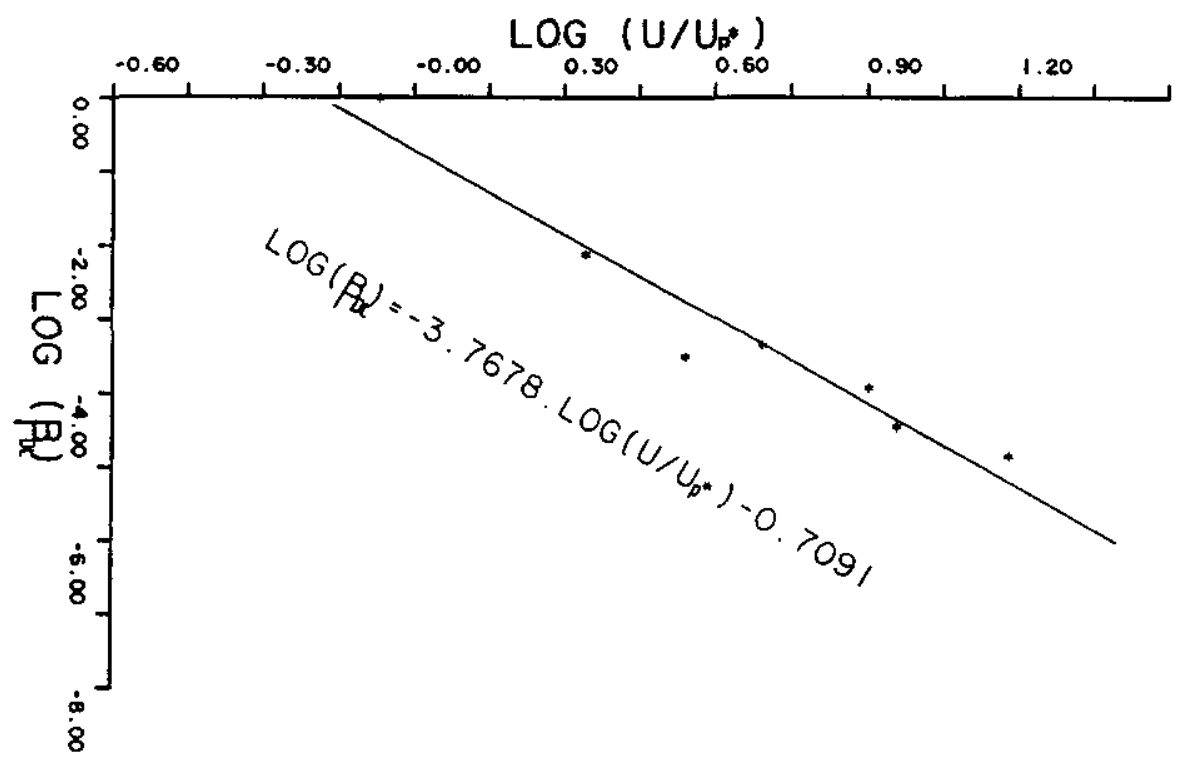

Figure 7.- Relations entre $B D L$ et $\mathrm{U} / \mathrm{U}^{*}$ pour les 2 tronçons considérés. Figure 7.- Relations between $B D L$ and $U / U^{*}$ in the tio stretches considered in the Albenche river. 
Figure 8.- Simulation du transport du matérlel dissous dans 1'Albenche (TO : 15.08 .83 à $22 \mathrm{~h}$ )

Les valeurs experimentales ( $\star$ ) se trouvent dans les plans $x=0.150 \mathrm{~km}$ (arrière plan de la figure, station amont) et $x=3.200 \mathrm{~km}$ (premier plan de la figure, station aval).

Figure 8.- Simulation of the dissolved material transport in the Albenche river (TO: 15.08 .83 at 22h)

The experimental values (*) are plotted on the background plane of the picture $(x=0.150 \mathrm{~km}$, upstream station) and on the foreground plane of the picture $(x=3.200 \mathrm{~km}$. downstream station).

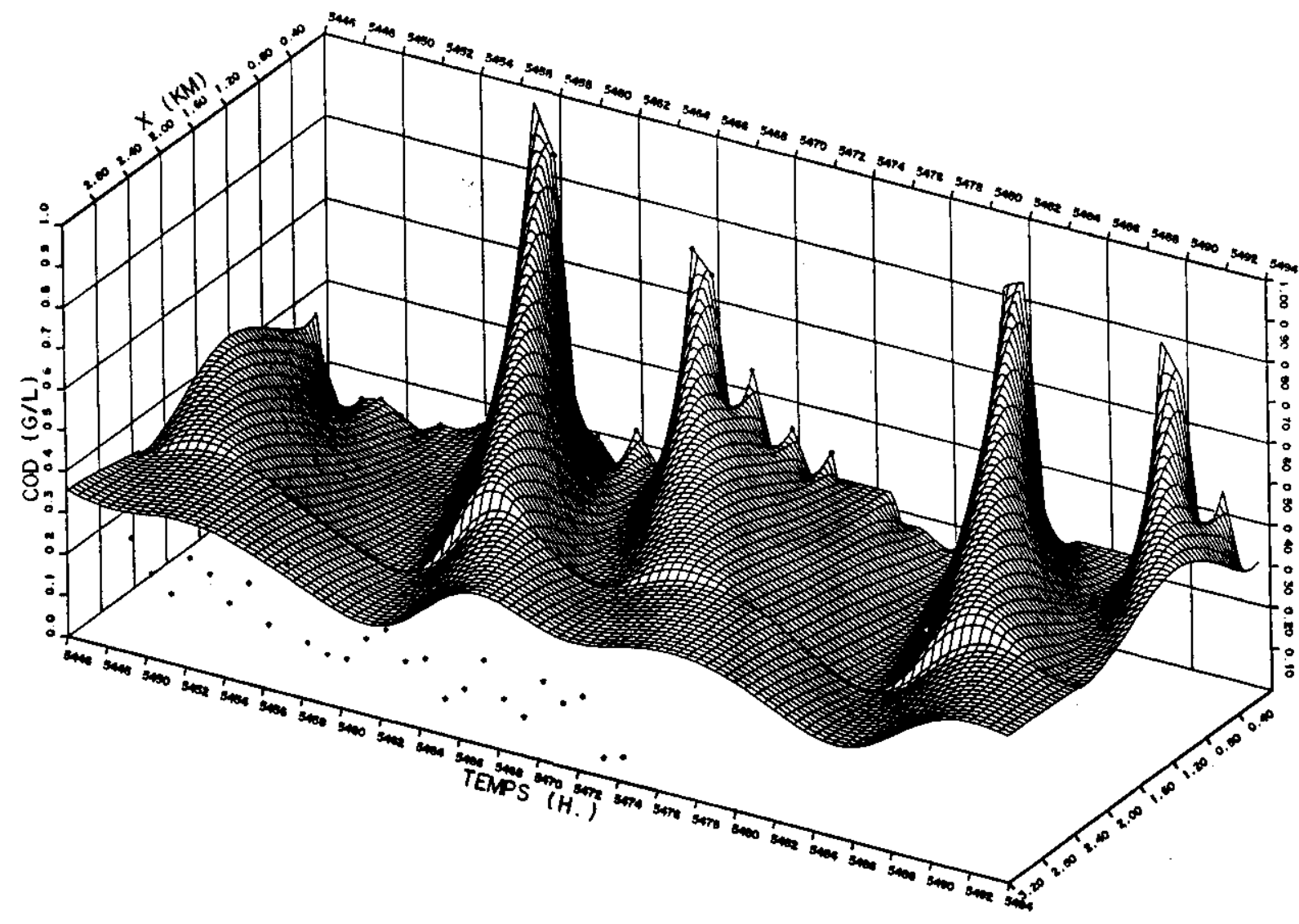


Figure 9. - Simulation du transport du matériel dissous dans 1'Albenche (TO : $04.10 .83 \mathrm{a} 18 \mathrm{~h}$ )

Les valeurs experimentales $(*)$ se trouvent dans les olans $x=0.150 \mathrm{~km}$ (arrière plen de la figure, station amont) et $x=3.200 \mathrm{~km}$ (premier plan de la figure, station avall.

Figure 9." Simulation of the dissolved material transport in the

Albenche miver

(TO: 04.10 .83 at $18 \mathrm{~h}$ )

The experimental values ( $)$ are plotted on the background plane of the picture $(x=0.150 \mathrm{~km}$, upstream station) and on the foreground plane of the picture $(x=3.200 \mathrm{~km}$, downstream station).

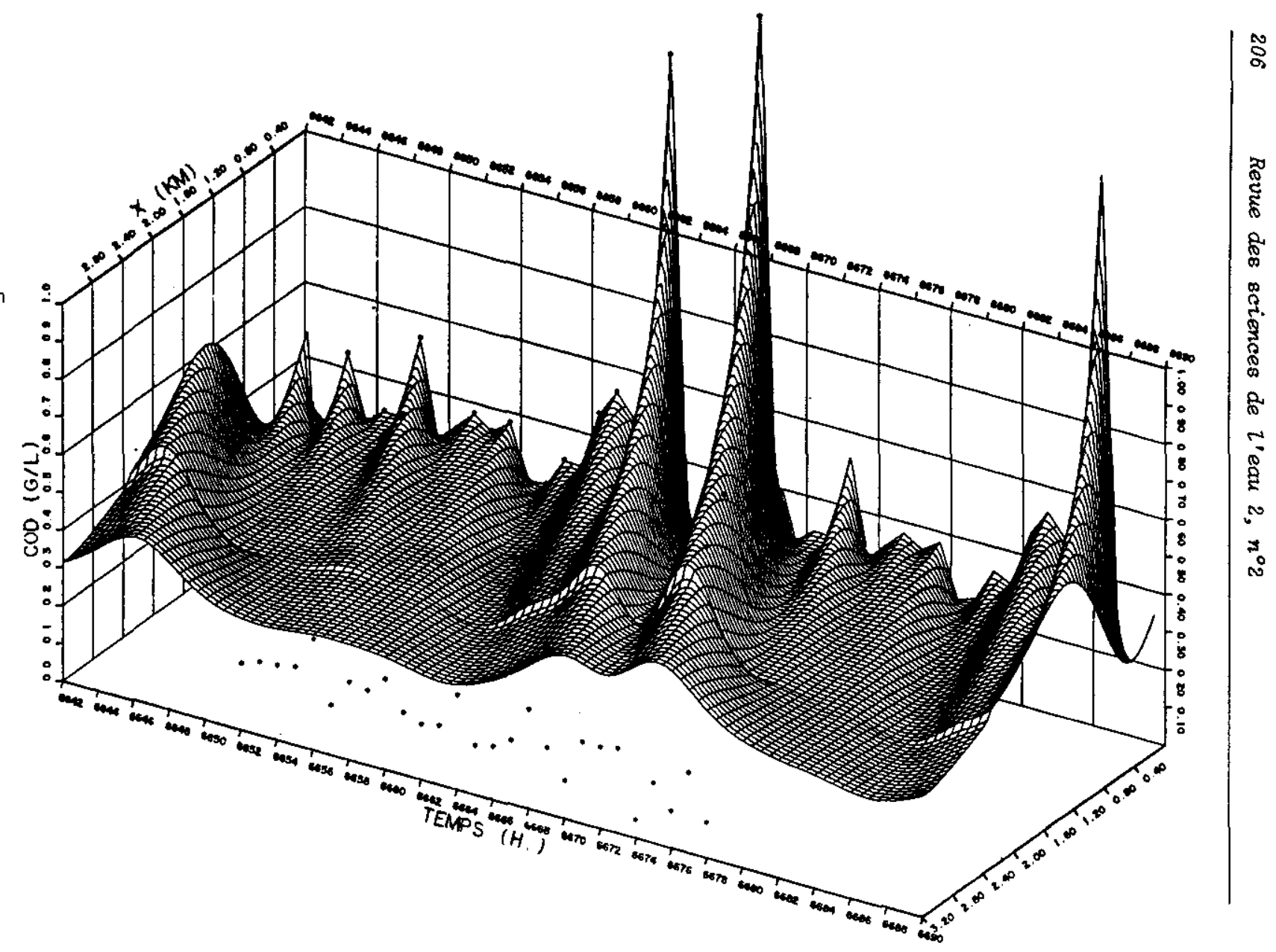


La prise en compte de ces deux types d'approche, débouche sur des modèles dont la structure mathématique est relativement complexe (systèmes d'équations aux dérivées partielles). Cette complexité pose de nombreux problèmes lors de la mise en oeuvre du modèle, notamment lors de 1'identification des paramètres. Une partie de ces difficultés peuvent etre levées si l'on considère la faible influence, dans certains cas tel que celui de l'Albenche, des processus blologiques sur les mécanismes hydrophysiques. Cette constatation autorise le découplage de la mise au point des équations décrivant la partie physique de celle des équations décrivant la partie bjologique du modèle. L'ajustement des nombreux paramètres du modèle global en est facilité.

Le but de ce premier travail, était de souligner l'importance des mécanismes hyärophysiques, notamment des mécanismes de transport, et de caler la partie hydrophysique du modẻle à l'aide d'expérience de traçage.

En ce qıi concerne les mécanismes hydrophysiques, la structure actuelle du modèle est satisfaisante mais pourrait encore être améliorée en prenant en compte, par exemple, les échanges entre l'écoulement et les zones mortes. On constate également, que dans un cours d'eau corme l'Albenche, la dispersion longitudinale a une grande importance. Elle explique le fort étalement du nuage de traçeur ou de polluant au niveau de la station aval. Elle influence donc les quantités de nourriture transportées par l'écoulement et disponibles pour les biocoenoses. Il faut soullgner l'importance de l'incorporation de ce mécanisme de dispersion dans les modèles du type de celui écrit ici pour l'Albenche car ce mécanisme est. très souvent négligé dans les modèles de petits cours d'eau.

Dans le cas du C.O.D., les valeurs experimentales sont expliquées partiellement par la dispersion longitudinale. La comparaison des résultats et des mesures suggère que 1'activité des biocoenoses benthiques est responsable de la disparition de plus de 35 o de la charge organique dissoute de l'eau de 15.08 (fig. 8) et de plus de 40 le 04.10 (fig. 9). Cette hypothèse sera examinée dans une prochaine publication concernant. le modèle biologique qui a êté couplé au modèle hydrophysique.

\section{RÉFÉRENCES BIBLIOGRAPHIQUES}

BAJRAKTAREVIC-DOBRAN H. (1982). DIspersion in mountainous natural streams. $J$. environ. Eng., 108(3): 502-514.

BECK M.B. (1985). Water quality management : a review of the development and opplication of mathematical models. Springer-Verlag, Berlin, 112 p..

BECK M.B., Van STRATEN G. (1983). Uncertainly and forecasting of water quality, springer-Verlag, Berlin, 386 p..

BEDFORD K.W., SYKES R.M., LIBICKI C.

(1983). Dynamic advective water quality model for rivers. $J$, enviror. Eng., $109(3)$ : 535-554.

BENCALA K.E., WALTERS R.A. (1983). Simulation of solute transport in a mountain pool-and-riffle stream : a transient. storage model. Water Resour. Res, 19(3): 718-724.

BENCAIA K.E., RATHBUN R.E., JACKMAN A,P. (1983). Rhodamine WT dye losses in a mountain stream environment. Water Resour. Bull., 19(6): 943-950. 
BIWAS A.K. (1981). Models for water quality monagement. Mac Graw-Hill, New-York, 348 p. .

BOLING R.H., GOODMAN E.D., Van STCKLE J.A., ZIMMER K.W., CUMMINS K.W., PETERSEN R.C., REICE S.R. (1975a). Toward a model of detritus processing in a woodland stream. Ecology, 56: 141-151.

BOLING R.H., PETERSEN R.C., CUMMINS K.w. $(1975 b)$. Ecosystem modeling for small woodland streans. In PATTEN B.C. (ed.), Systems onalysis and simulation in ecology, vol. III. Acadenic Press, New-York, 183-204.

BOYLE J.D., SCOTT J.A. (1984). The role of benthic films in the oxygen balance in a east Devon river. Water Res., 18(9): 1089-1099.

BUJON G. (1983). Modélisation de la dispersion des substances solubles ou pseudo-solubles dans un cours d'eau. Application au cas de la seine à l'amont de Paris. La Houille Blanche, 1: 45-53.

CANALE R.P. (1976). Modeling biochemical processes in aquatic ecosystems. Ann Arbor Sclence, Ann Arbor, 389 p..

CAUSSADE B., chaussavotne c., Masberatat L. (1982). Modelling of turbulent fluxes in water surface ecosystems studies. Mathematics and Computers in Simulation, 24: 224-229.

CAZELLES B. (1987). Modélisation d'un ecosystème lotique : dynamique du carbone organique dissous et des microorganismes berthiques dans un cours d'eau pollue. Thès $=$ de Doctorat $n^{\circ} 6887$, Université Lyon I, 427 p..

CLESCERI L.S., PARK R.A., BLOMFIELD J.A., (1977). General model of microbial growth and decomposition in aquatic ecosystems. App 2. environ. Microbiol., 33: 1047-1058.

DUBOIS D.M. (1981). Progress in ecological engineering and management by mathematical modelling. Editions CEBEDOC, Liège, 1024 p..

DE ANGELIS D.L., GOLDSTEIN R.A., O'NEIL R.V. (1975). A model for trophic interaction. Ecology, 56: 881-892.

ELDER J.W. (1959). The dispersion of maxked fluid in turbulent shear flow. $J$. Fluid Mechanics, 5: 544-560.

FABRE J., RAYANY BALA P. (1980). Equations générales de transfert de masse, de quantite de mouvement, d'énergie, dans les eaux de surface. Annls univ. Abidjan, Série C, Tomes XVI: 70-102.
FISCHER H.B. (1964). Determination of dispersion coefficients by change of moment method. Technical memorandum, N 64-6, California institute of technology, Pasadena.

FISCHER H.B. (1967). The mechanics of dispersion in natural streams. $f$. hydraul. Div., 93: 187-216.

FISCHER H.B. (1968). Dispersion predictions in natural streams. J. Sanit. Eng., 94: 927-941.

FISCHER H.B. (1973). Longitudinal dispersion and turbulent mixing in open channel flow. Annual Rev. Eluid Mechonics, 5: $59-77$.

FONTVIEILLE D. (1987). La circulation du carbone organique dans les écosystèmes lotiques : cas du phénomène d'autoépuration. These de Doctorat d'etat $n^{\circ} 8747$, Université Lyon $I, 189$ p..

FONTVIEILLE D., CAZELlES B. (1985). Modification de l'importance relative des flux de carbone organique d'un ruisseau pollué aprés une période de crues. Verh. internat. Verein. Limol., 22: 2270-2276.

FONTVIEILLE D., CAZELLES B. (1988) Seasonal changes of some physiological and structural parameters in the sediments of an organically polluted stream. Verh. internat. Verein. Limol., 23: 1306-1312.

FONTVIEILLE D., RENAUD M. (1982). A method of estimating the respiration of mud communities in shallow running water. Water Res., 16, 5: 593-599.

HALL C.A.S., DAY J.W. (1977). Ecosystem modelling in theory and practice : an introduction with case histories. wiley, New-York, 684 p..

hARPEMOES P. (1982). Immediate and delayed oxygen depletion in rivers. Water Res., 16: 1093-1098.

HUFF D.D., O'NEIL R.V., EMANUEL W.R., ELWCOD J.W., NEWBOLD J.D. (1982). FlOW wariability and hillslope hydrology. Earth Surface Processes and Londforms, 7: 91-94.

HVITVED-JACOBSEN T. (1982). The impact of combined sewer overflows on the dissolved oxygen concentration of a river. Water Res., 16: 1099-1105.

INNIS G.S., O'NEIL R.V. (1979). Systems analysis of ecosystems. International Cooperative Publishing House, 425 p..

JARRETT R.D. (1984). Hydraulics of highgradient streans. J. Hydraul. Eng., 110 , 11 : $1519-1539$. 
JORGENSEN S.E. (1979). State of the art in ecological modelling. Pergamon press, oxford, 891 p..

JORGENSEN S.E. (1983). Application of ecological modelling in environmental monagement, part A. Development in envirommental modelling 4. Elsevier, Amsterdan, 722 p..

JORGENSEN S.E. (1986). Fundamentals of ecological modelling. Development in envirormental modeliting 9. Elsevier, Amsterdam, 289 p..

JORGENSEN S.E., MITSCH W.J. (1983). Application of ecological modelling in environmental management, part $B$. Development in environmental modelling 4. Elsevier, Amsterdam, 438 p..

LAUENROTH W.K., SKOGERBOE G.V., FLUG M. (1983). Analysis of ecological systems : state-of-the-art in ecological modelling. Development in environmental modelizing 5. EIsevier, Amsterdan, 920 p..

LEOPOLD L.B., WOLMAN M.G., MILLER J.P. (1964). Fluvial processes in geomorphology. Freeman, San Francisco, 522 p..

LIU H. (1977). Predicting dispersion coefficients of stream. J. environ. Eng., 103: 56-69.

LIU H., CHENG A.H.D. (1980) , Modified fi.ckian model for predicting dispersion. J. hydroul. Div., 106(6): 1021-1040.

LOCK M.A. (1981). River epilithon : a hight and organic energy transducer. In LOCK M.A. \& WILIIAMS D.D. (eds), Perspectives in muning water ecology. plenum Press, New-York, p. 3-40.

LOCK M.A., WALLACE R.R., COSTERTON J.W., VENTULLO R.M., CHARLTON S.E. (1984). River eplitthon : toward a structuralfunctional model. Oikos, 42: 10-22.

MaC INTIRE C.D., COLBY J.A. (1978). A hiexarchical model of lotic ecosystems. Ecol. Monogr., 48(2): 167-190.

MITSCH W.J., BOSSERMAN R.W., KLOPATEK J.H. (1981). Energy and ecological modelling. Development in ecological modelling 1. Elsevier, Amsterdam, 639 p..

NEWBOLD J.D., ELWOOD J.W., O'NEIL R.V., SHELDON A.L. (1983). Phosphorus dynamics in a woodland stream ecosystems : a study of nutrient spiralling. Ecology, 64(5): $1249-1265$.

O'NEIL R.V., ELWOOD J.W., HILDEBRAND S.G., (1979a). Theoretical implications of spatial heterogeneity in stream ecosystems. In INNIS G.S. O'NEIL R.V. (eds). Systems conatysis of ecosystems. International cooperative Publishing touse, p. 79-102.
ORLOB G.T. (1981), Models for stratified impoundments. In BISWAS A.X. (ed.), Models of water quality management. Mac Graw-Hill, New-York, p. 273-313.

PATTEN B.C. (1975). Systems analysis and simulation in ecology, Vol. III. Academic Press, New-York, 601 p..

RINALDI S., SONCINI-SESSA R., STEHFEST H., TAMURA н. (1979). Modelling and control of river quality. Mac Graw-Hill, New-York, 380 p..

SABOL G.V., NORDIN C.F. (1978), Dispersion in rivers as related to storage zones. J. hydraul. Div., 104, 5: 695-708.

SAYRE พ.พ. (1973a), Natural mixing processes in riviers. In SHEN H.W. (ed.), Environmental impact on mivers, river Mechanics III. Fort collins, Collorado, p. $6,1-6,37$.

SCAVIA D., ROBERTSCN A. (eds). (1979). Perspectives on lake ecosystem modeling. Ann Arbor Science, Ann Arbor, 326 p..

SOMLYODY L. (1982), Water-quality modelling : a comparison of transportoriented approaches. Ecol. Modelling, 17: $183-207$,

SRINANTHAKUMAR S., AMIRTHARAJAG A. (1983). Organic carbon decay in stream with bioflim kinetics. J. Environ. Eng., 109, 1: 109-119.

STRASKRABA M., GNAUCK A. (1985). Freshwater ecosystems, modelling ond simulan tion. Developments in environmental modelling 8. Elsevier, Amsterdam, 309 p.

THERIEN N., MORRISON K.A., COUPAL B. (1981). The impact of reservoin flooding on a freshwater benthic community. In MITSCH W.J., BOSSERMAN R.W. \& KLOPATEK J.M. (eds), Energy and ecological modelling. Elsevier, Amsterdam, p. 249-259.

VALEANTINE E.M. \& WOOD I.R. (1977), Longitudinal dispersion with dead zones. J. Hydraul. Div., 103: 975-990.

WEBSTÉR J.R. (1983). The role of benthic macrolnvertebrates in detritus dynamics of streams : a computer simultation. Ecol. Monogr., 53, 4: 383-404.

WEBSTER J.R., BENFIELD E.F., CAIRNS J. Jr (1979). Model predictions of the effects of impoundment on particulate organic matter transport in a river system. In WARD J.V. STANFORD J.A. (eds), The ecology of regulated streams. Plenum Press, New-York, p. 339-364.

WUHRMANN $K$. (1972). Stream puxification. In MITCBELL R. (ed.) : Water pollution microbiology. Wiley, New-York, p. 119 151. 